\title{
Structure of shock waves in particulate composites
}

Cite as: J. Appl. Phys. 127, 065902 (2020); https://doi.org/10.1063/1.5125449

Submitted: 23 August 2019 . Accepted: 25 January 2020 . Published Online: 12 February 2020

M. B. Rauls, and G. Ravichandran (iD)
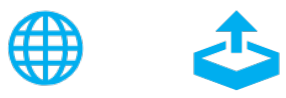

View Online

Export Citation

CrossMark

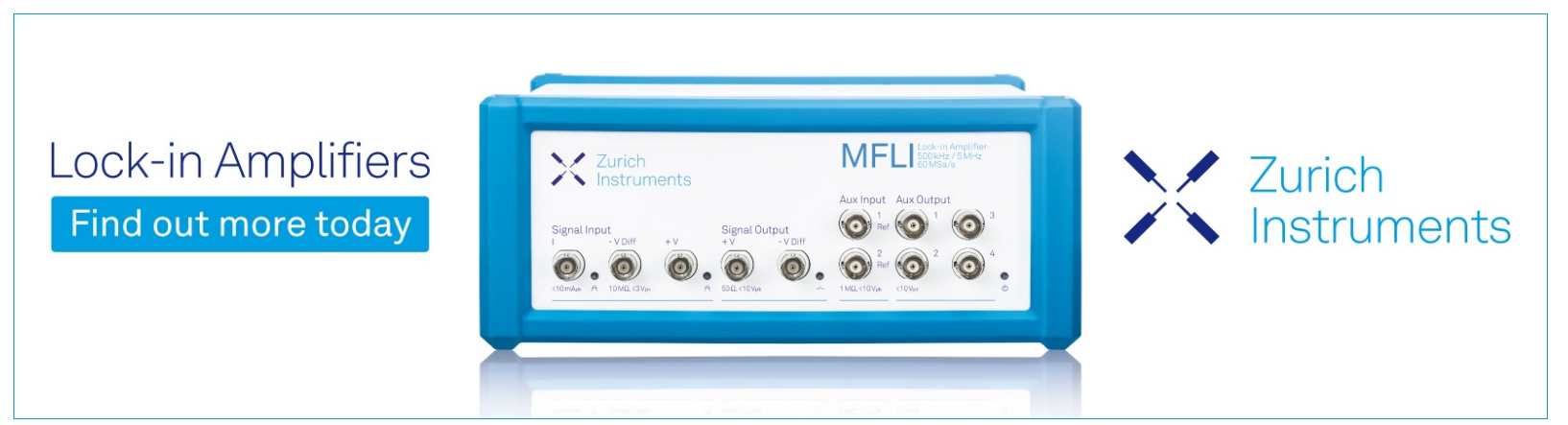




\title{
Structure of shock waves in particulate composites
}

\author{
Cite as: J. Appl. Phys. 127, 065902 (2020); doi: 10.1063/1.5125449 \\ Submitted: 23 August 2019 . Accepted: 25 January 2020 . \\ Published Online: 12 February 2020
}

M. B. Rauls and G. Ravichandran ${ }^{\text {) }}$ (iD

\author{
AFFILIATIONS \\ Division of Engineering and Applied Science, California Institute of Technology, Pasadena, California 91125, USA
}

a) Author to whom correspondence should be addressed: ravi@caltech.edu

\begin{abstract}
The shock compression response of particulate heterogeneous solids was investigated using normal plate impact experiments and numerical simulations. A model composite system of silica glass spheres embedded in a matrix of thermoplastic polymer, polymethyl methacrylate, was developed to mimic the impedance mismatch of structural and energetic heterogeneous materials. Shock wave profiles were measured at multiple points on the rear surface of the composite specimens to characterize shock dispersion and spatial heterogeneity in material response due to the random distribution of particles. Composites with single mode as well as bimodal bead diameter distributions were subjected to plate impact loading at $\sim 1000 \mathrm{~m} / \mathrm{s}$ resulting in an average shock stress of $\sim 4 \mathrm{GPa}$. Shock rise times were measured for composites of $30 \%$ and $40 \%$ glass by volume, with spherical particles of diameters in the range of $100-1000 \mu \mathrm{m}$. In the case of single mode composites, the shock wave rise times were observed to scale linearly with particle diameter divided by the bulk shock wave speed. The addition of a second bead size to a base size in a $30 \%$ glass by volume composite mix resulted in significant increases in shock rise time. Numerical simulations were used to develop insights into scattering and the development of shock structure in particulate composites. Shock disruption mechanisms due to particles and matrix/interface damage effects are discussed.
\end{abstract}

Published under license by AIP Publishing. https://doi.org/10.1063/1.5125449

\section{INTRODUCTION}

Structures designed for habitation, protection, and transportation may be subjected to shock, impact, and blast loading, both intentionally and unintentionally. Such events subject materials to high pressures and cause deformation at extremely high strain rates. ${ }^{1,2}$ The bulk of construction and infrastructural materials are highly heterogeneous, and hence, it is important that the dynamic response of such materials as a function of their level of heterogeneity is well characterized and understood. Heterogeneous materials such as concrete are characterized by the presence of hard, high modulus particulate inclusions embedded in a relatively compliant matrix. Other materials that share these characteristics in common use are potting compounds for electronic devices and energetic materials such as high explosives. When the high interface densities of concrete and polymer bonded explosives (PBX) are considered, it is clear that wave reflections and scattering at interfaces dominate the development of the shock wave structure. ${ }^{3}$

Shock disruption in composites is typically characterized by the degree of departure of a straight planar shock front from its assumed form of a propagating sharp discontinuity in stress and particle velocity. In order to mitigate the damage from shock loading, a strategy is sought to optimally disrupt shock propagation by increasing the rise time, thus decreasing the strain rate and particle acceleration. At present, there is relatively little understanding of the role of microstructure in heterogeneous materials on shock front disruption and particle acceleration. Scattering and wave reflections are among the dominant mechanisms of shock front disruption in heterogeneous materials such as composites. ${ }^{3,4}$ Optimal scattering is said to be achieved when the inclusion phase (particles) disrupts the straight shock front as it propagates across the sample, resulting in the largest possible rise time of the steady structured shock. The magnitude of shock disruption is highly dependent on the length scale of the heterogeneity, material properties, and the shock strength. At moderate and low levels of shock amplitude, the shock structure observed is mostly dependent on the amplitude, as lower magnitude shocks in composite materials are not typically steady.

Zhuang et al. employed a simple method of introducing a known spatial heterogeneity into a shock experiment using alternating layers of homogeneous materials impacted normal to the 
layer interface planes. ${ }^{4}$ The final steady shock profile that they observed was dependent on the number of interfaces, as well as the shock impedance mismatch at the interface. It was also found that an increase in impedance mismatch between the layers resulted in increased scattering and longer rise times. Increasing interface density reduced the observed rise time and increased the amplitude and frequency of oscillations. Mean heterogeneity size was identified as a dominant length scale in the scattering of shock waves. Their experiments also revealed that the strain rate associated with the shock front is proportional to the square of the shock stress in layered composites. This is in contrast to the Swegle-Grady relationship observed in nominally homogeneous materials such as metals and ceramics where the strain rate is proportional to the fourth power of the shock stress.

Using theoretical modeling, Molinari and Ravichandran modeled stress-strain rate dependence for shocks in both homogeneous ${ }^{6}$ and heterogeneous materials. They found that when a stress wave interacts with an interface, some of the energy is reflected, transmitted, or dissipated by interface damage or friction. Grady ${ }^{8}$ summarized the dependence of stress on the strain rate in a variety of different metals, ceramics, and heterogeneous materials. Strain rate associated with steady shock fronts in periodic layered heterogeneous materials scale with the second power of the shock stress, indicating a much greater shock "viscosity" in comparison to homogenous materials. ${ }^{4-8}$ The impedance mismatch of the layers reduces the material acceleration at the shock front by redirection of energy. The shock profile becomes steady as a balance is reached between primary reflections sending energy away from the shock front and secondary reflections catching up through precompressed media behind the shock front.

In contrast to periodic layered media, global constructive and destructive interference modifying the shock wave structure is not the sole mechanism in particulate composites. Local response and geometry and distribution of particulates also become important factors in the overall shock response of such materials. Shock wave studies of particulate composites may be divided broadly into two categories: (i) those driven to high enough pressures to propagate structured steady waves and (ii) those at lower pressures that develop structured waves but are dominated by matrix response. Setchell and Anderson ${ }^{9}$ suggested that at low pressures, the observed wave front is characterized by a "lazy S" shaped curve, which is dominated by the viscoplastic response of the matrix. At higher pressures, the wave front develops into a shock front that propagates as a structured steady wave. Most of the work in the literature on heterogeneous solids is focused on the Hugoniot response as a function of impedance mismatch and particulate size. ${ }^{10-21}$ Notable composites include concretes, polymer bonded explosives, epoxy-ceramics, epoxy-metal, and polymer-ceramics. These materials cover a large range of impedance mismatch ratios and particulate sizes. An overview of the data available in the literature is presented in Fig. 1, which provides the range of particle sizes and impedance mismatch. Except for the smallest particulate sizes studied in the literature, the particle size distributions have been largely polydisperse. The volume fraction of second phase particulates is typically around $40 \%-55 \%$ beyond which agglomeration becomes an issue.

The studies undertaken on structural grades of concrete focus on specimens with particulate size in the range of tens of $\mathrm{mm} .^{18}$

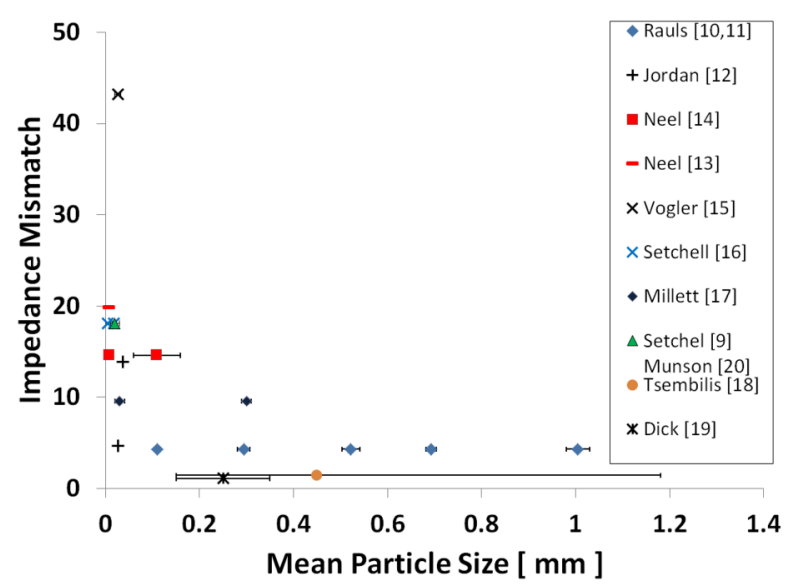

FIG. 1. Summary of particle size vs impedance mismatch ratio for various particulate composites that have been subjected to shock compression. Adapted from Refs. 9-20.

Polymer bonded explosives (PBX) also fall into the range of materials where scattering induced by particulates is a significant mechanism that defines the shock wave structure. Simulations and experiments undertaken with the PBX samples showed strong strain rate dependence on the shock structure. ${ }^{22}$

Epoxy matrix based potting compounds are typically found in shock protection applications for electronics. The potting composites in common use are composed of epoxy with finely ground alumina, tungsten carbide, and aluminum. Typical particle sizes are on the order of $1-100 \mu \mathrm{m}$, and the impedance mismatch ratios in these composites are typically in the range of $30-50,{ }^{14,15}$ which is much greater than those of concretes and high explosives. A study of alumina particle-based composites with a lower impedance mismatch ratio was undertaken by Neel and Thadhani ${ }^{13,14}$ to determine the effect of particle size on shock response. The alumina particles in the tetrahydrocannabivarin (THV) polymer-based composite were separated in to 1,10 , and $100 \mu \mathrm{m}$ nominal diameters. The Hugoniot state response appeared to be independent of particle size, but stress time profiles showed a dependence on particle size. Numerical simulations have been used to simulate multiphase materials including particulate composites ${ }^{23}$ and granular materi$\mathrm{als}^{24}$ to understand the role of microstructure and geometry on shock properties. ${ }^{25}$ The simulations have primarily focused on the Hugoniot and the equation of state (EoS) of heterogeneous materials and the shock structure has received relatively less attention. Preliminary experimental observations of the current study were presented earlier in a conference proceedings. ${ }^{10}$ The shock structure in particulate composites is systematically explored in greater detail in this paper, and numerical simulations to develop insights into the mechanisms of scattering due to particles are presented.

The present study focuses on developing an understanding of the development of the structure of steady shocks in particulate composites and their dependence on particle size. The model particulate composite, its fabrication process, mechanical properties, and numerical models, parameters, and geometries are described in 
Sec. II. The experimentally observed dependence of shock structure on particulate volume fraction and mean diameter is examined, and a quantitative statistical representation of the degree of shock front disruption is presented and discussed in Sec. III. The summary and conclusions for the study are presented in Sec. IV.

\section{MATERIALS AND METHODS}

\section{A. Materials}

The model composite material chosen for the study serves as a simulant for engineered composite materials of interest such as concrete and energetic materials. To achieve the desired impedance mismatch of 2-10 between the matrix and the particle, a common industrial polymer, polymethyl methacrylate (PMMA), is chosen as the matrix material. ${ }^{11}$ Spherical glass beads in various graded sizes are chosen as the particle reinforcement. PMMA may also be heat treated to relieve or take advantage of residual stress introduced in the molding process to control interface properties. Composites can be made with strong, perfect transparent interfaces or thermally shocked to produce weaker interfaces.

An even, random distribution mimics the common composites of interest and is also readily amenable to simulations on geometries with randomly generated particulate distributions. A PMMA polymer powder of $75000 \mathrm{MW}$ (P/N 04553, Polysciences, Warrington, PA) with a glass transition temperature $\left(T_{g}\right)$ of $105^{\circ} \mathrm{C}$ is used to serve as the matrix for the particulate composites in this study. The measured density of the as molded and heat-treated polymer is $1180.4 \pm 0.3 \mathrm{~kg} / \mathrm{m}^{3}$. Porosity was found to be low and the molded PMMA had a density of $99.5 \%$ of commercially available cast acrylic material $\left(1186 \mathrm{~kg} / \mathrm{m}^{3}\right)$. Bulk elastic wave speeds were measured from as molded samples using ultrasonic wave reflection experiments. A 5000 series (Olympus, Waltham, MA) pulser-receiver and accompanying transducers were used to measure the time of flight of waves passing through PMMA disks of known thickness. The elastic longitudinal and shear wave speeds were determined to be 2680 and $1350 \mathrm{~m} / \mathrm{s}$, respectively. Standard values for density $(\rho)$ and longitudinal wave $\left(C_{l}\right)$ speed for the particulate material, silica glass, are $2203 \mathrm{~kg} / \mathrm{m}^{3}$ and $5930 \mathrm{~m} / \mathrm{s}$, respectively. ${ }^{29}$ The longitudinal elastic impedances (product of density and longitudinal wave speed) of PMMA and glass are computed to be 3.163 and $13.064 \mathrm{MPa} / \mathrm{m} / \mathrm{s}$, respectively, resulting in an impedance mismatch ratio of 4.13 between the reinforcement phase and the matrix.

Molding parameters for the 75000 MW PMMA used in this study are readily available. ${ }^{26,27}$ The as obtained glass beads are sorted using stainless steel sieves (Grainger, Los Angeles, CA) of appropriate size to obtain a monodispersed collection of beads of the desired size. Based on the desired volume fraction of glass inclusions, appropriate weights of PMMA powder and glass are combined and mixed in an analog vortex mixer (VWR, Radnor, PA). They are then immediately poured on a polished flat base platen in a mold tube (30 mm diameter) made of 304 stainless steel that was preheated. Once the mold is loaded with the well-mixed powder and glass beads, a punch is forced down as far as it will go to lock the powder down and keep it from shifting during the remainder of the fabrication process. The clearance between the diameters of the mold cavity and punch platen was designed to

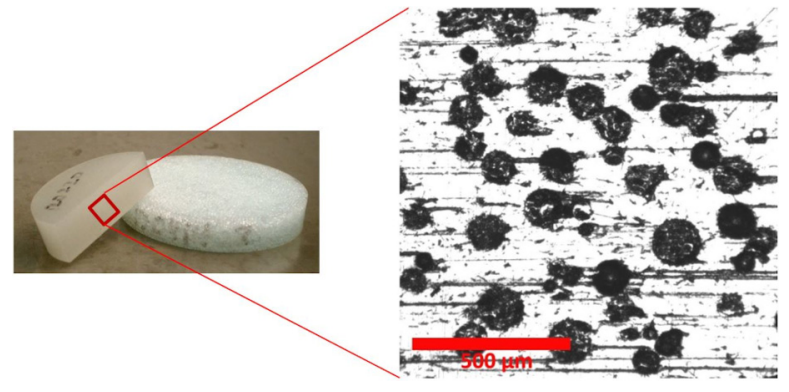

FIG. 2. Compression molded $30 \mathrm{~mm}$ diameter PMMA-glass (22\% volume fraction) composite specimen and a micrograph of the cross section showing the particulate distribution. Reproduced with permission from Rauls and Ravichandran, Proc. Eng. 103, 515 (2015). Copyright 2015 Elsevier.

minimize PMMA flash while still permitting air to escape from the mixture. The assembled mold is then transferred to a vacuum bag, which is evacuated to at least 635 Torr $(-25 \mathrm{in} . \mathrm{Hg})$, and the mold is placed between the compression mold platens and a $2670 \mathrm{~N}$ (600 lbf.) force is applied. Once the pressure is applied, the heaters in the platens are turned on and allowed to heat up to $180^{\circ} \mathrm{C}$. The temperature and pressure were chosen to permit plastic flow around the glass spheres, without the polymer becoming so fluid that the glass beads can settle. After a period of $18 \mathrm{~h}$, the mold is removed and allowed to cool gradually in an oven and subjected to a two-step heat-treating process to relieve internal stresses and maintain a strong bond between the phases of the composite. ${ }^{23}$

The samples with strong bonding are nearly transparent, and the interfaces are difficult to see. A compression molded composite specimen (22\% glass by volume) and a micrograph of the cross section are shown in Fig. 2. The typical composite samples are $30 \mathrm{~mm}$ in diameter and $7.1 \mathrm{~mm}$ in thickness.

\section{B. Experimental setup}

Normal plate impact experiments were conducted using a $36 \mathrm{~mm}$ smooth bore breach loading powder gun in the Graduate Aerospace Laboratories at Caltech (GALCIT) with a barrel length of approximately $3 \mathrm{~m}$. Impact velocities attainable with this system range from 200 to $2000 \mathrm{~m} / \mathrm{s}$. A four-channel heterodyne velocimeter system, commonly known as the photonic Doppler velocimeter $(\mathrm{PDV}){ }^{28}$ is used for measuring projectile and particle velocities. Projectile velocity is measured during experiments with one of the channels of the PDV system, while the impact time is recorded with a set of four electric shorting pins (CA-1038, Dynasen, Goleta, CA) flush mounted with the target. The tilt of the flyer with respect to the target is computed from the impact times measured by the shorting pins. The free surface particle velocities at three different locations on the rear surface of the sample are measured using the PDV and are used to assess the spatial heterogeneity of the shock wave profiles. The PDV signals are recorded using an Agilent (Santa Clara, CA) MSO oscilloscope (bandwidth, $4 \mathrm{GHz}$; sampling rate, $20 \mathrm{GSa} / \mathrm{s}$ ). Parameters such as the shock width, $10 \%-90 \%$ rise time, and shock speed are then computed and 
averaged from the multiple points measured for each target. Standard deviations are calculated for each measured quantity to offer insights into the precision confidence of the value. The details of the experimental setup can be found in Ref. 11.

\section{Numerical simulation}

Sandia National Laboratories' CTH shock hydrocode simulation software ${ }^{29,30}$ was used for experimental design and analysis of the PMMA and glass bead particulate composites. CTH is a combined Eulerian-Lagrangian solver, which is widely used to simulate the propagation of stress waves in solids and fluids. A twodimensional model of the three-dimensional plate impact problem was implemented in CTH. A center "section" view was selected to give a rectangular domain with a vertical dimension given by the plate diameter and the horizontal dimension given by the plate thickness. Once the bead sizes and locations are determined, they are inserted into the PMMA matrix. An aluminum flyer plate is then defined in the up-range direction and given an initial down range velocity, i.e., impact velocity. The top and bottom of the geometry (the radial free surfaces of the target plate) have a defined boundary condition to minimize wave reflections. The mesh used in the simulations has a pitch of $20 \mu \mathrm{m}$, giving at least five cell widths across the smallest bead in the study. A convergence study was undertaken by increasing the spatial resolution with smaller nodal pitches but did not offer any additional improvement.

Simulation geometries were chosen to match the experimental cases as closely as possible and match the dominant length scale and volume fraction. Creating a two-dimensional analog of a threedimensional composite requires some assumptions about the conversion between volume fraction and area fraction. It has been shown that the area fraction of inclusions on a plane through a material of interest with a sufficiently random distribution is equivalent to the volume fraction of particulates in the whole sample. ${ }^{31}$ The area fraction alone is not enough to determine the number of beads to be inserted in each simulation geometry. Since the objective of the study was to determine the scaling of rise time with respect to some characteristic length scale, the diameter of the beads inserted in the two-dimensional geometry was chosen to match the diameter of the beads present in the experimental samples. A Gaussian distribution for bulk bead diameter distribution was assumed. The micrographs of samples showed an evenly dispersed random arrangement of beads within the PMMA matrix (Fig. 2). Consequently, friction at interfaces is neglected and particles are assumed to be perfectly bonded to the matrix. Bead center locations were also generated with two independent uniform random variables.

Sample geometries for a single mode simulation and a bimodal simulation are shown in Fig. 3. The empty rectangle to the left of the composite samples shown is occupied by aluminum and is given an initial velocity corresponding to the experiment that is being simulated. The aluminum flyer plate in the simulations was $7 \mathrm{~mm}$ thick and extends past the left-hand field of view in Fig. 3. The full geometry mimics the experimental target diameter to preclude release waves from the edges reaching the tracer locations during shock break out and for sufficient time afterward to measure the structure of the shock wave and the Hugoniot state.
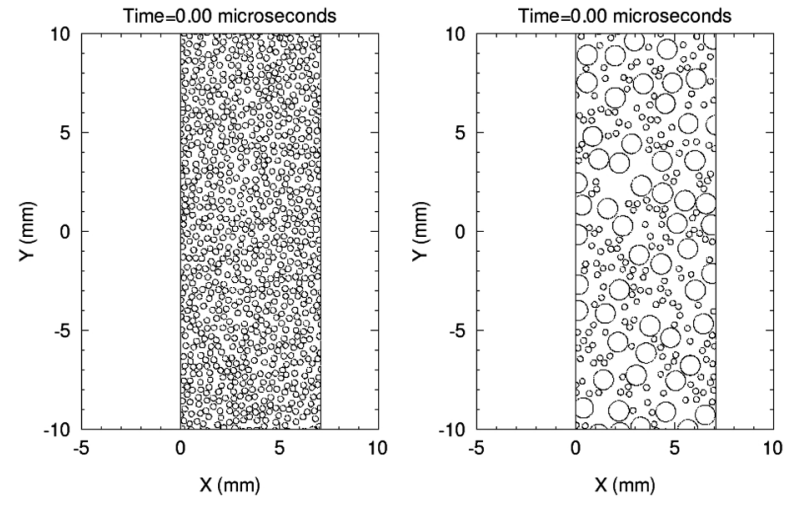

FIG. 3. Sample simulation geometries of the PMMA/glass particulate composite samples for CTH: $300 \mu \mathrm{m}$ beads (left) and $1000 \mu \mathrm{m}$ beads with $300 \mu \mathrm{m}$ interstitials (right).

Silica glass is a well-characterized material, and a significant body of experimental data exists on its response at high pressures. The pressures of interest in the present study tend to be at the lower range of those studied. The use of a SESAME tabular EoS allows for the most general representation of the material. ${ }^{32}$ Both the PMMA and aluminum use a separate primary EoS: MGRUN. ${ }^{33}$ The Hugoniot data have been fit to the Mie-Grüneisen equation of state $^{2}$ and the Grüneisen function, $\Gamma$, is defined as the dependence of pressure $(P)$ on internal energy $(E)$ at constant density $(\rho)$,

$$
\Gamma=\rho^{-1}\left(\frac{\partial P}{\partial E}\right)_{\rho} .
$$

The Grüneisen parameter is only a function of density and the pressure is given by

$$
P(\rho, E) \approx P_{H}(\rho)+\Gamma(\rho) \rho\left[E-E_{H}(\rho)\right] .
$$

The implementation of the Grüneisen formulas in the simulations assumes that the specific heat, $C_{V}$ (used for temperature calculations), is constant. The values of $P_{H}$ and $E_{H}$ fall on the measured material Hugoniot $(H)$. So long as the study remains within the pressure ranges explored by previous experiments that were used to fit the Hugoniot, the MGRUN model offers a rapid, accurate computation of state variables. The parameters used in the models for the relevant materials are given in Table I.

Material strength models were also implemented for all materials in the simulation. A viscoelastic plastic (VEP) model was used for PMMA, a Steinberg-Guinan (SG) model was used for aluminum, and a user defined model was for the glass. The VEP model implemented in CTH is based on a parallel Maxwell model. ${ }^{29}$ VEP is capable of resolving both quasistatic material behavior as well as rate dependent viscoplasticity via parallel Maxwell elements. A shear "spring" with an initial modulus is employed to capture the low strain rate response. Up to five parallel Maxwell elements (consisting of a series spring and dashpot) are available in the model. The quasistatic shear modulus is represented by the quantity $G_{0}$. Subsequent 
TABLE I. Mie-Grüneisen model parameters ${ }^{29}$ for $6061-T 6$ aluminum and PMMA used in simulations.

\begin{tabular}{lcccc}
\hline \hline Material & $\begin{array}{c}\text { Density, } \\
\rho_{0}\left(\mathrm{~kg} / \mathrm{m}^{3}\right)\end{array}$ & $\begin{array}{c}\text { Bulk sound } \\
\text { speed, } C_{0}(\mathrm{~m} / \mathrm{s})\end{array}$ & $\begin{array}{c}\text { Hugoniot } \\
\text { slope, } s\end{array}$ & $\begin{array}{c}\text { Grüneisen } \\
\text { parameter, } \Gamma\end{array}$ \\
\hline $\begin{array}{l}6061 \\
\text { Aluminum }\end{array}$ & 2703 & 5200 & 1.4 & 1.97 \\
PMMA & 1186 & 2600 & 1.5 & 0.97 \\
\hline \hline
\end{tabular}

parameters $G_{n}$ and $\mu_{n}$ in the Maxwell elements are calibrated to experimental data at various strain rates so that the model is applicable to as many loading cases as possible. A separate set of VEP coefficients is available for each of the components of the deviatoric stress. The VEP model is also capable of accumulating damage and updating the material properties and maximum available shear resistance. Deviatoric strain rates are allowed to approach zero as the material becomes fully damaged, but it can still support some shear stress when loaded in hydrostatic compression after damage. The constitutive relation relating shear stress $(\tau)$ and shear strain rate $\left(\dot{e}_{v e}\right)$ for each of the Maxwell elements is

$$
\begin{gathered}
\tau_{0}^{\prime}=2 G_{0} \dot{e}_{v e}, \\
\tau_{m}^{\prime}=2 G_{m}\left(\dot{e}_{v e}-\frac{\tau_{m}}{\mu_{m}}\right) .
\end{gathered}
$$

The stress rates are integrated with an Euler method to determine stress as a function of time. The material model inputs of the two-element $(m=1,2)$ Maxwell model for PMMA are presented in Table II.

The flyer plate material 6061-T6 aluminum was described using a Steinberg-Guinan-Lund (SGL) viscoplastic model. This model takes into account strain hardening, strain rate dependency, and pressure dependent shear strength. ${ }^{29}$ A Poisson's ratio of 0.17 and a general failure surface were used for the glass, as no significant plasticity is to be expected in such brittle materials at the level of stress considered in this study.

A validation study of the model parameters built into $\mathrm{CTH}$ in describing the shock response of the PMMA was performed. Three experiments on pure molded PMMA without particulates were performed at nominally 800,1000 , and $1200 \mathrm{~m} / \mathrm{s}$ impact speeds with a 6061-T6 aluminum flyer. The free surface velocity profiles obtained

TABLE II. Viscoelastic plastic (VEP) model parameters ${ }^{29}$ for PMMA used in simulations.

\begin{tabular}{cc}
\hline \hline Quantity & Value \\
\hline Density & $1186 \mathrm{~kg} / \mathrm{m}^{3}$ \\
Poisson's ratio & 0.4 \\
Quasistatic shear modulus, $G_{0}$ & $1000 \mathrm{MPa}$ \\
Shear modulus, $G_{1}$ & $300 \mathrm{MPa}$ \\
Shear modulus, $G_{2}$ & $250 \mathrm{MPa}$ \\
Damping coefficient, $\mu_{1}$ & $300 \mathrm{~Pa} \mathrm{~s}$ \\
Damping coefficient, $\mu_{2}$ & $40 \mathrm{~Pa} \mathrm{~s}$ \\
\hline \hline
\end{tabular}

using the PDV are shown in Fig. 4. Solid traces are the measured shock response, while the dashed traces are the simulation data. Other than a tracer location artifact (a small blip) on the leading edge of the simulation data, the time of arrival, steady state particle velocity, and shock wave structure are very similar.

The measured Hugoniot of pure PMMA from the experimental data (Fig. 4),

$$
U_{s}=2786+1.329 u_{p}
$$

with shock $\left(U_{s}\right)$ and particle $\left(u_{p}\right)$ velocities in $\mathrm{m} / \mathrm{s}$ compares reasonably well with the Mie-Grüneisen model parameters (bulk sound speed, $C_{0}$, and slope of the Hugoniot, $s$ ) in Table I, which are based on the data from Vogler et al. ${ }^{21}$ The measured Hugoniot of PMMA also agrees reasonably well with more recent experimental data in the literature from the work of Lacina et al. $\left(C_{0}=2550 \mathrm{~m} / \mathrm{s}\right.$ and $\left.s=1.54\right) .{ }^{34}$ Some of the differences in the values for bulk sound speed $\left(C_{0}\right)$ and slope of the Hugoniot $(s)$ between those presented here can be attributed to the range of particle velocities over which the Hugoniot is considered. The Hugoniot $\left(U_{s}-u_{p}\right)$ for PMMA is nonlinear ${ }^{25}$ and is approximated by a linear relationship in the region of interest.

A series of experiments guided by the simulations were undertaken for single mode bead diameter distribution composites comprised of $30 \%-40 \%$ glass by volume. The initial simulation results were used to determine the appropriate bead diameter range for experimental observation, as well as to assist in the classification of shock front disruption regimes. Shock front disruption is quantified from measured wave profiles for both monodispersed and bimodal bead diameter distribution composites. An analysis of the limitations of the use of simple shock hydrocodes for composite analysis is presented.

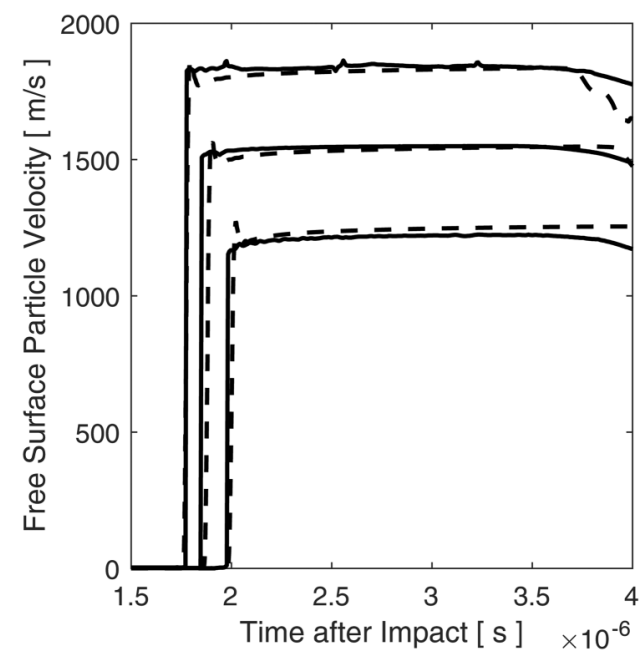

FIG. 4. Simulation of shock wave experiments on pure PMMA for validation of the VEP model. Solid traces are from plate impact experiments at impact velocities of $781 \mathrm{~m} / \mathrm{s}, 1011 \mathrm{~m} / \mathrm{s}$, and $1211 \mathrm{~m} / \mathrm{s}$, and the dashed traces are from numerical simulations at corresponding impact velocities. 
A parametric study was undertaken to determine the scaling of the shock front disruption, as measured by the observed shock wave rise time on the rear free surface of a particulate composite subjected to planar plate impact. Composite mixtures of $30 \%$ and $40 \%$ glass particles by volume were impacted at a velocity of approximately $1000 \pm 10 \mathrm{~m} / \mathrm{s}$ by a $7 \mathrm{~mm}$ thick 6061-T6 aluminum alloy flyer plate. Nominal stresses of approximately $4 \mathrm{GPa}$ were imposed over a long pulse duration. Monodisperse beads were obtained at five discrete average bead diameters, for a total of ten individual shock wave experiments. Three separate wave profiles were obtained per experiment using PDV to determine the level of spatial heterogeneity and provide error bounds on the measured shock rise times. Initial CTH calculations were performed to plan the ideal bead diameters to explore in the experiments.

\section{RESULTS}

\section{A. Simulations}

Model and geometry parameters for the simulation of a sample with randomly placed particulates were presented in Sec. II. The randomly generated microstructures are subjected to a $1000 \mathrm{~m} / \mathrm{s}$ impact by a 6061-T6 aluminum flyer at time $t=0$. Bead diameters were chosen to cover the particle size range prescribed by composites of interest. Five cases for simulation were chosen to determine if there was a strong dependence of rise time on average bead diameter for monodisperse bead distributions. Based on manufacturing constraints observed in preliminary studies, composites of $30 \%$ glass by volume and $40 \%$ glass by volume were simulated.

Spatial heterogeneity can be considered to increase, as the mean bead diameter increases away from the characteristic length scale given by the shock width in pure PMMA. In the case of a $1000 \mathrm{~m} / \mathrm{s}$ impact with a 6061-T6 aluminum flyer plate, the shock width in pure PMMA was measured to be $22.96 \pm 0.77 \mu \mathrm{m}$. This value is based on the measured steady shock speed of $3.826 \pm 0.128 \mathrm{~mm} / \mu \mathrm{s}$ and an observed rise time of $\sim 6 \mathrm{~ns}$. The uncertainties reported for the shock width and shock speed are based on PDV measurements at three locations on the free surface. The average bead diameters chosen span a range of two orders of magnitude from the unaltered shock width, offering adequate room for experimentation. Hydrostatic pressure contour plots were generated with $\mathrm{CTH}$ at $1 \mu \mathrm{s}$ after flyer plate impact. The results for $100 \mu \mathrm{m}, 500 \mu \mathrm{m}$, and $1000 \mu \mathrm{m}$ diameter mean bead size at $40 \%$ glass by volume are shown in Fig. 5 .

It is clear from Fig. 5 that the imposed shock wave has traversed approximately the same distance into each composite sample, with the primary difference then being the thickness and morphology of the shock front. At the smallest average bead diameter of $100 \mu \mathrm{m}$, the bead size is not significantly different from the shock thickness in pure PMMA as seen in Fig. 5 (left). A roughly planar thin shock front is observed, even with a large number of interfaces present. Many reflections are noted in the shock loaded region of the PMMA and glass composite. At this scale, it is difficult to observe secondary reflections that redirect scattered energy back toward the shock front. After a short distance (less than $0.5 \mathrm{~mm}$ ), the shock width stabilizes to be roughly equal to the average bead size.

As the mean bead size is increased to $500 \mu \mathrm{m}$ in Fig. 5 (center), rippling and broadening of the shock front are noted. The shock front is rippled approximately evenly across its length, and the steady state shock width again approaches the mean bead diameter of the composite. There are sufficiently many beads and interfaces present to ensure that the shock does not encounter uninterrupted regions of PMMA. Pressure concentrations are observed due to constructive interference of stress waves in the PMMA binder. These large differences in stresses likely lead to large shear stress levels, especially near the interfaces. No immediate opening of voids is observed, but the simulations are not specifically intended to visualize or predict damage in these composites.

The $1000 \mu \mathrm{m}$ glass bead composite simulation in Fig. 5 (right) again follows the same trend of widening the shock front to near the mean bead diameter. A shock front this wide greatly reduces
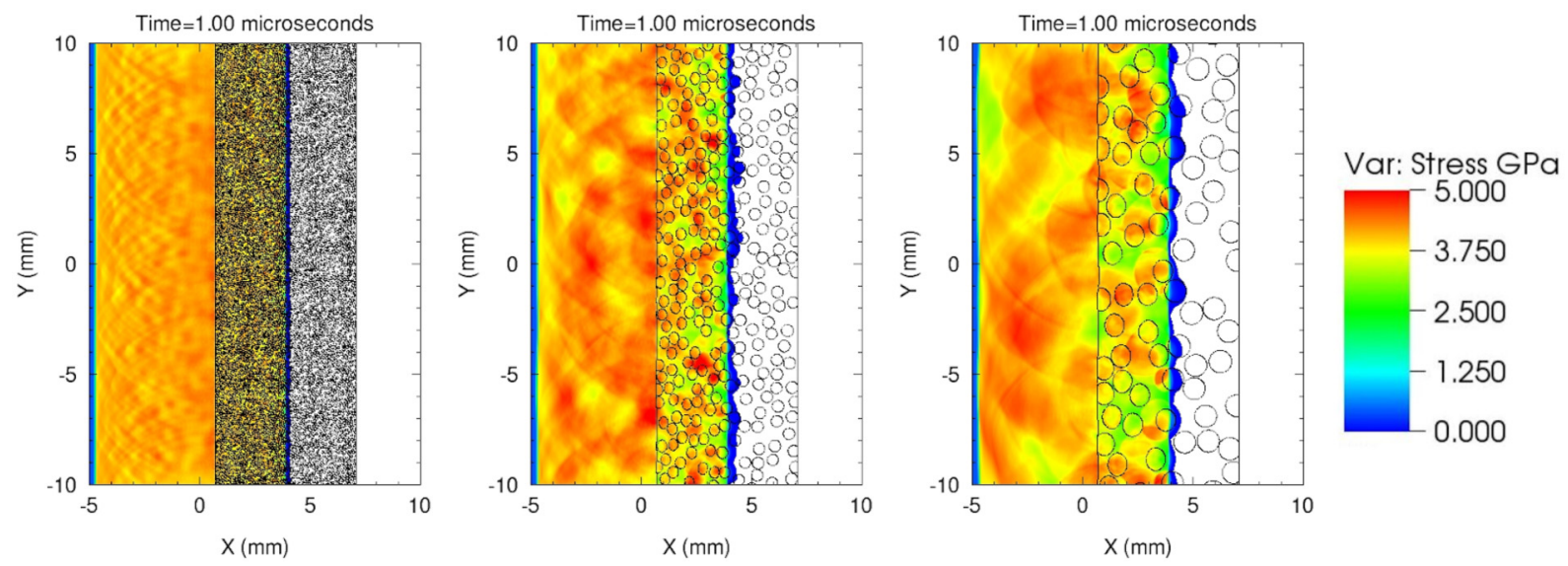

FIG. 5. Simulation results for pressure contours in GPa at $1 \mu \mathrm{s}$ after impact for $40 \%$ volume fraction of $100 \mu \mathrm{m}$ (left), $500 \mu \mathrm{m}$ (center), and $1000 \mu \mathrm{m}$ (right) glass beads in a PMMA matrix. Impactor is $6061-\mathrm{T} 6$ aluminum with an impact velocity of $1000 \mathrm{~m} / \mathrm{s}$. The shock thickness (blue contour) is comparable to the bead diameter evolves to become nonplanar as the particle size increases due to the disruption of the shock front. 
particle accelerations and thus the forces seen by the material at the shock front. The limit to this behavior at a fixed glass volume fraction is that there are relatively fewer beads and interfaces present to serve as scattering sites. This manifests itself in nonoptimal scattering, where significant areas of the disrupted shock front can straighten out. In Fig. 5 (right), consider the horizontal paths at $y=0.5 \mathrm{~mm}$ and $y=4 \mathrm{~mm}$. At $y=0.5 \mathrm{~mm}$, a very narrow shock front is approaching a glass bead at $x=4 \mathrm{~mm}$. The wave has already passed through a bead at $x=1.5 \mathrm{~mm}$, but the wave front transits through pure PMMA after the first bead allows for the front to straighten out. The multipoint velocity measurement capability discussed in Sec. II is ideal for quantifying the level of spatial heterogeneity upon shock breakout on the rear surface. It is expected that a large uncertainty in measured shock particle velocity rise times could exist.

The morphology of the rear surface as a function of time from the simulations was also examined. The location of the rear surface was tracked at discrete times after shock breakout (ASB) in order to quantify rippling and spatial disruption. Based on the above discussion of shock front disruption as it propagates through the material, there should be a difference in the ripple magnitude and period on the rear surface. The ripple period will not be constant due to the randomness inherent in the composite geometry. Figure 6 illustrates the surface deformation over a $5 \mathrm{~mm}$ line centered on $y=0$, i.e., centerline of the specimen.

When the composites are made of small beads, the magnitude of the surface ripples are small, and the pitch, or peak to peak distance, is small, as seen in Fig. 6 (left) for the $100 \mu \mathrm{m}$ beads. This rippling is approximately $10 \mu \mathrm{m}$ in amplitude with a pitch of approximately $200 \mu \mathrm{m}$. The fact that neither of these scales appears related to the mean bead diameter indicates that optimal scattering has not been achieved and that the beads of diameter similar to the shock width in pure PMMA do not have a dramatic effect on shock thickness. The surface disruption reaches a steady state around $30 \mathrm{~ns}$ after shock breakout (ASB). A definite change in rear surface morphology is observed with larger beads.

In the $500 \mu \mathrm{m}$ bead diameter case shown in Fig. 6 (right), a steady rear surface profile develops at approximately $144 \mathrm{~ns}$ ASB. The ripple at this time is approximately $50-75 \mu \mathrm{m}$ in amplitude.
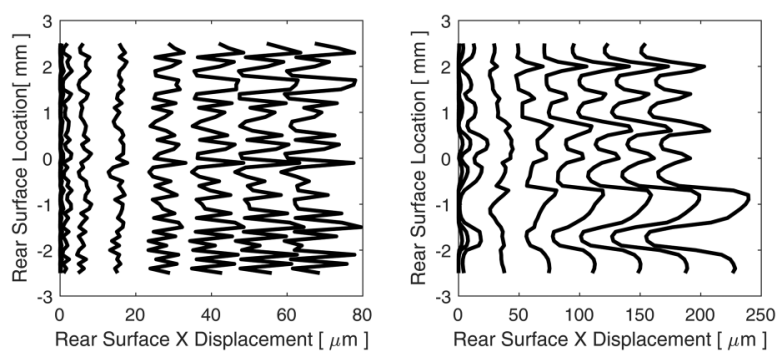

FIG. 6. Rear surface morphology from simulations after shock breakout at the free surface for $40 \%$ volume fraction of $100 \mu \mathrm{m}$ (left) and $500 \mu \mathrm{m}$ (right) glass beads in the PMMA matrix composite. Surface profiles progress by $10 \mathrm{~ns}$ from left to right in the $100 \mu \mathrm{m}$ bead composite and by $24 \mathrm{~ns}$ in the $500 \mu \mathrm{m}$ bead composite.
Pitch of the ripples is near the mean bead diameter at $500 \mu \mathrm{m}$. This shape is most likely due to the placement of the beads near the rear surface in the area of interest. Studies performed on different geometries of beads placed in regular grid arrangements (face centered and close packed hexagonal grids) returned ripple pitches related to the vertical spacing of the rows of the beads, a quantity determined by the volume fraction of glass present, not the bead size. While surface morphology is a reasonable measure of spatial disruption, using it as a scale factor for shock modification is not advisable. At best, it gives a hint to the size of the uncertainty bounds that must be placed on the metric used to quantify shock front modifications.

The CTH simulations were successful in determining that there are multiple scattering effectiveness regimes across the range of available bead sizes. The bead sizes span one order of magnitude and the corresponding shock thickness can be 10-100 times the shock width in pure PMMA. These composites can also be realized through the processing method described in Sec. II.

\section{B. Experiments}

Planar plate impact experiments with monodisperse bead diameter distributions centered about the mean used in the simulations were conducted using glass beads embedded in the PMMA matrix as described in Sec. II. Rear surface wave profiles for $30 \mathrm{~mm}$ diameter and $7.1 \mathrm{~mm}$ composite plates impacted at $1000 \mathrm{~m} / \mathrm{s}$ by 6061-T6 aluminum flyer plates were measured using multichannel PDV. The PDV fiber probes had an outer diameter of $1.8 \mathrm{~mm}$ with a collimating lens, which resulted in a spot size of $0.4 \mathrm{~mm}$ in diameter on the target. In order to eliminate the target plate thickness as a variable and ensure the validity of the shock jump relations, a series of shots was completed with composite target plates of varying thickness before proceeding with varying the particle size. The experimental parameters (particle size, volume fraction of glass beads, impact velocity), measured experimental results (shock speed, particle velocity, rise time), and computed quantities (shock stress, strain rate) for all the experiments are presented in Table IV in the Appendix.

\section{Examination of steady shocks: Hugoniot and viscosity}

Three experiments were undertaken to confirm that the shock waves observed after passing through $7.1 \mathrm{~mm}$ of composite target plates were fully developed and steady when observed. $100 \mu \mathrm{m}$ and $500 \mu \mathrm{m}$ glass beads at $40 \%$ by volume were used in the fabrication of these target plates. Three experiments for each of the bead sizes with different plate thicknesses were conducted. All shots were completed with impact velocities of $1010 \pm 5 \mathrm{~m} / \mathrm{s}$ except for the $7.1 \mathrm{~mm}$ thick target with $100 \mu \mathrm{m}$ beads, which was impacted at $1078.4 \pm 1.1 \mathrm{~m} / \mathrm{s}$. Glass beads on the smaller side of the spectrum were also chosen to minimize the risk of measuring geometry specific effects, such as the case of measuring rear surface particle velocity in a bead free region. Experimental results showing steady wave profiles are shown in Fig. 7. The target plate thicknesses examined are $5 \mathrm{~mm}, 7.1 \mathrm{~mm}$, and $10 \mathrm{~mm}$. The choice of $100 \mu \mathrm{m}$ and $500 \mu \mathrm{m}$ beads enables the use of shock structures to verify the level of steady wave development. In Fig. 7, there is a significant short duration particle velocity overshoot present above the late 

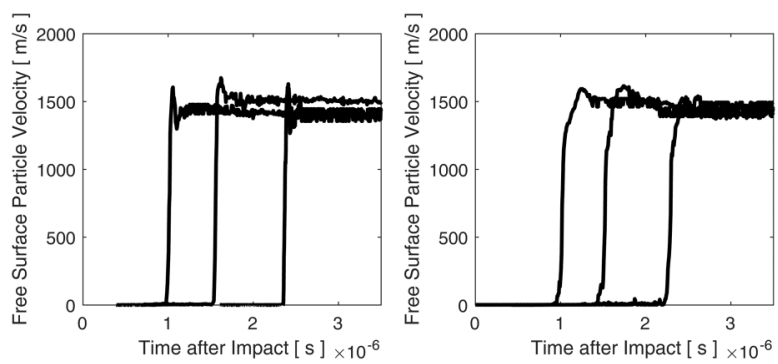

FIG. 7. Steady wave profiles observed for $40 \%$ volume fraction $100 \mu \mathrm{m}$ (left) and $500 \mu \mathrm{m}$ (right) glass beads in the PMMA matrix composite. Target thicknesses increase from $\sim 5 \mathrm{~mm}$ to $7.1 \mathrm{~mm}$ to $10 \mathrm{~mm}$ in each trace.

time response for the $100 \mu \mathrm{m}$ bead samples. The $7.1 \mathrm{~mm}$ target shows a higher steady state particle velocity due to its higher impact velocity, while the initial transient width and relative height are similar. Transit time (impact occurs at approximately $-0.2 \mu \mathrm{s}$ ) is commensurate with the target plate thickness.

The development of shock widths much greater than the shock width in pure PMMA warrants the examination of development time to steady state at larger bead diameters as well. The $500 \mu \mathrm{m}$ diameter bead composites show similar shock profile development characteristics, Fig. 7 (right). The wave profile is nearly identical for each thickness case, and the wave speeds in each case match closely.

In order to evaluate steady wave profile development distance, ${ }^{35}$ knowledge of the Hugoniot properties of the PMMA and composite material are required. Targets with $40 \%$ volume fraction $500 \mu \mathrm{m}$ glass beads that are $7.1 \mathrm{~mm}$ thick were fabricated and impacted by aluminum flyer plates at velocities between 580 and $1400 \mathrm{~m} / \mathrm{s}$. Shock speeds were computed based on the difference of the average time of arrival as measured by tilt pin shorting times and the $10 \%$ particle velocity level of the shock. The data for shock speed as a function of particle velocity from the experiments are plotted in Fig. 8 for the PMMA/glass composite and pure PMMA matrix.

Referencing Fig. 8, the linear approximation of the Hugoniot for the $500 \mu \mathrm{m}$ bead at $40 \%$ glass by volume composite can be written as

$$
U_{S}=3508+0.809 u_{p},
$$

where the particle velocity $\left(u_{p}\right)$ and shock speed $\left(U_{s}\right)$ are expressed in $\mathrm{m} / \mathrm{s}$. It is interesting to note that the slope $(s)$ of the Hugoniot is smaller for the $40 \%$ glass composite (0.809) in comparison with the corresponding value for the matrix, PMMA [1.329, Eq. (5)].

It is important to ensure that the shock structure has fully developed by the time it reaches the rear surface so that the scaling law developed for shock disruption is meaningful. Bland ${ }^{35}$ determined that an approximate required shock propagation distance for attaining steady state, $\delta$, can be written as

$$
\delta=\frac{3}{8} \frac{C_{0}}{s} \frac{1}{\dot{\varepsilon}}
$$

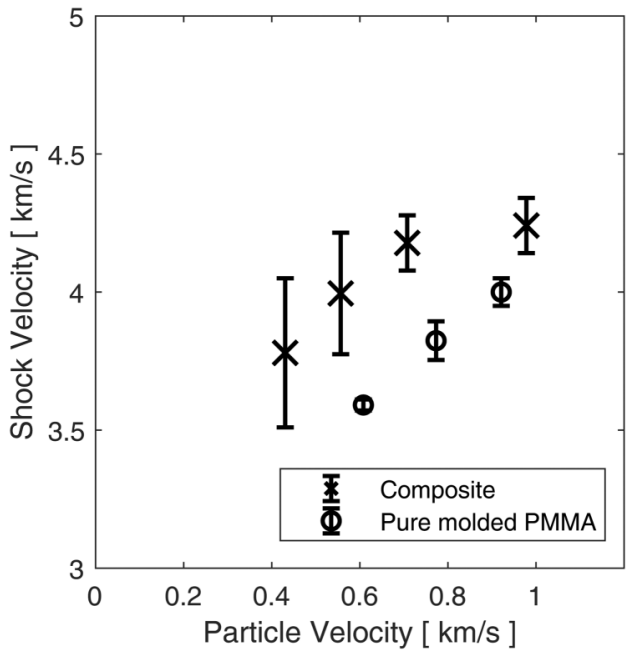

FIG. 8. Shock speed vs particle velocity Hugoniot data for PMMA/glass composite with $40 \%$ volume fraction $500 \mu \mathrm{m}$ glass beads and pure PMMA.

where $C_{0}$ and $s$ are the parameters in the linear Hugoniot shock speed vs particle velocity relationship. The strain rate, $\dot{\varepsilon}$, can be approximated by the jump in strain divided by the rise time, $\tau$, both of which are readily available from experimental measurements,

$$
\dot{\varepsilon}=\frac{u_{p}}{U_{S} \tau} \text {. }
$$

Substituting Eq. (8) into Eq. (7),

$$
\delta=\frac{3}{8} \frac{C_{0}}{s} \frac{U_{S} \tau}{u_{p}}
$$

The characteristic distance, $\delta$, is then computed using Eq. (9) to be $1.76 \mathrm{~mm}$ at an impact velocity of $\sim 1000 \mathrm{~m} / \mathrm{s}$. Within the experimental error, the shocks appear to be steady and the structure fully developed. Constant shock speed combined with a qualitative structure analysis indicate that the steady wave assumption made in the theoretical analysis holds in the case of these particulate composites.

By using the rise time of the shock front and the shock speed $\left(U_{s}\right)$ (see Table IV in the Appendix), the strain rate [Eq. (8)] was computed from the shock front profiles for the $500 \mu \mathrm{m}$ bead at $40 \%$ glass by volume composite. The experiments resulted in shock stress amplitude in the range of $2.25-6.38 \mathrm{GPa}$, which was computed using the reflected shock Hugoniot for the impactor, which is made of 6061-T6 aluminum. It was observed that the strain rate scales as the square of the shock stress, which is similar to the scaling law found for other heterogeneous materials including layered solids ${ }^{4,7,8}$ and granular materials. ${ }^{21}$ It would be interesting to examine whether this or similar scaling law indicating higher viscosity for particulate composites hold for other particle sizes and volume fractions. 


\section{Shock wave profiles}

Five average bead diameters were chosen, which were nominally the same to those used in the $\mathrm{CTH}$ simulations. The average diameters and their bounds are given in Table III. A uniform distribution about the mean is assumed. Due to the tightness of the bounds, the beads used in each composite can be considered to be monodisperse. A series of ten experiments consisting of five mean bead sizes (100, $300,500,700$, and $1000 \mu \mathrm{m})$ and two bead volume fractions $(30 \%$ and $40 \%$ ) was planned. Due to the anticipated spatial heterogeneity in the shock, three points on the rear surface of each target were illuminated with collimating PDV probes. A particle velocity time history for each point on the rear free surface was recorded.

Figures 9 and 10 show rear surface particle velocity signals, with time $t=-0.2 \mu$ s corresponding to time of initial impact on the composite target by the aluminum flyer. The probes are located on the circumference of a $5 \mathrm{~mm}$ diameter circle, coincident with center of the target disk. Probes are located at 4, 8, and 12 o'clock positions on the circle. This placement ensures that the observation is not influenced by edge unloading waves until after the initial wave pulse reaches the rear surface. A $3 \mu$ s recording window was adequate for the purposes of the experiments.

At the smallest bead sizes, the slope of the shock wave is very large, indicating a very narrow shock thickness and a high strain rate. There is also a significant short duration overshoot above the steady state particle velocity. As the mean bead size increases, the slope of the leading wave pulse decreases, and the structure begins to develop at the leading edge and near the point of maximum particle velocity. This rounding is a significant part of the measured shock width, as the slope, while reduced, remains relatively steep.

In addition to the reduction in slope, the wave profile changes shape from a large short duration overshoot (Fig. 9), to a lower magnitude longer duration overshoot [Fig. 10 (left)], to a final fully rounded shape [Fig. 10 (right)]. In smaller bead diameter composites, the high interface count results in a large amount of primary reflections directing energy in the direction opposite to shock propagation. These primary reflected waves have a high likelihood of encountering another PMMA/glass interface, resulting in a secondary reflection directing energy back toward the shock front. As waves in compressed media travel faster than those propagating into a quiescent material, they may catch up with the leading shock pulse, contributing to the amplitude and short duration of the overshoot. As the bead diameter is increased, the constant volume fraction constraint enforces that there are fewer beads present, and thus fewer interfaces to redirect energy. The beads still serve as effective scatterers, thickening the shock front to near their average

TABLE III. Size of glass spheres used in composite fabrication and shock wave experiments.

\begin{tabular}{cc}
\hline \hline Label & Bead size $(\mu \mathrm{m})$ \\
\hline 100 & $110 \pm 6$ \\
300 & $294 \pm 13$ \\
500 & $522 \pm 19$ \\
700 & $693 \pm 12$ \\
1000 & $1006 \pm 25$ \\
\hline \hline
\end{tabular}
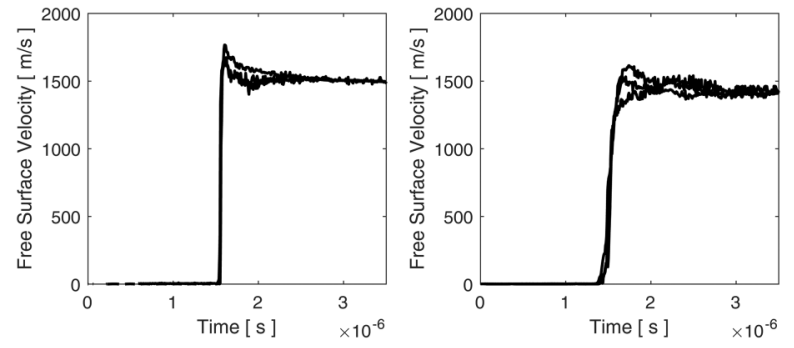

FIG. 9. Particle velocity vs time history for $40 \%$ volume fraction, $100 \mu \mathrm{m}$ (left) and $500 \mu \mathrm{m}$ (right) glass beads in the PMMA matrix composite. Impactor is $6061-\mathrm{T} 6$ aluminum with impact velocities $1078 \mathrm{~m} / \mathrm{s}(100 \mu \mathrm{m})$ and $1017 \mathrm{~m} / \mathrm{s}$ $(500 \mu \mathrm{m})$

diameter, but the magnitude of the overshoot is lessened, and the duration increased.

In order to verify that the overshoot observed is solely a product of the addition of scatterers into the PMMA matrix material, results from experiments on pure PMMA targets in Fig. 4 are examined. Both the shock profiles obtained experimentally do not exhibit any overshoot behavior, and the shock thickness remains around $20 \mu \mathrm{m}$. The slight bumps in the top part of the shock in the simulation results are an artifact of tracer position in regard to their placement on the rear surface and how CTH handles unloading across material cells. Once the boundary conditions are established, the particle velocity drops immediately back to the steady state velocity that is experimentally observed.

\section{Shock disruption: Rise time}

A quantitative metric is needed to evaluate the magnitude of shock disruption as a function of mean bead size in the composites of interest. In the field of control, models and controllers are evaluated in their response to step inputs by defining a rise time. In this study, a rise time is computed by measuring the time that it takes for the rear surface particle velocity to accelerate from $10 \%$ of its maximum value to $90 \%$ of its maximum value. The shock profile within the $10 \%-90 \%$ range contains information from both the
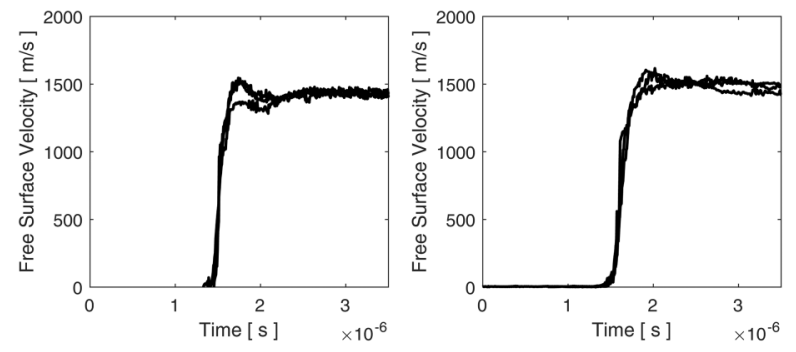

FIG. 10. Particle velocity vs time history for $40 \%$ volume fraction $700 \mu \mathrm{m}$ (left) and $1000 \mu \mathrm{m}$ (right) glass beads in PMMA matrix composite. Impactor is $6061-\mathrm{T} 6$ aluminum with impact velocities $1010 \mathrm{~m} / \mathrm{s}(700 \mu \mathrm{m})$ and $1012 \mathrm{~m} / \mathrm{s}$ $(1000 \mu \mathrm{m})$. 
change in the slope of the velocity time history, as well as the overshoot behavior. In order to account for the spatial heterogeneity, a rise time is computed for each probe pointed at the rear surface. An average is taken of the three spatially separated rise times, and a standard deviation is reported to give vertical error bounds. As the bead diameter is assumed to be uniform in between the bounds defined by the mesh screens, the horizontal error bars are set to these bounds. The experimentally observed rise times for 30\% and $40 \%$ glass beads by volume are plotted together with the simulation derived rise times in Fig. 11.

The observed rise times scale linearly with increasing particle diameter for composites with monodisperse bead size distributions. The shock width is increased by nearly an order of magnitude in both time and space between the smallest and largest particulates. For a given particle size, the rise time of the $40 \%$ glass by volume composite is larger than that for the $30 \%$ glass by volume composite. The inverse of the slopes of the linear best fit lines $\left(R^{2}=0.98\right)$ for the experimental data in Fig. 11, which have the units of velocity are $4.645 \mathrm{~mm} / \mu$ s and $3.877 \mathrm{~mm} / \mu$ s for the $30 \%$ and $40 \%$ glass by volume composites, respectively.

At low levels of heterogeneity (i.e., lower volume fractions and smaller bead diameters), the CTH model predictions for the rise time are closer to the values observed in experiments. However, there are significant differences between the experimental and simulated rise times for larger diameter beads and higher volume fractions. Interface integrity and local damage are not significant enough for the observed rise times to depart from the simulation results. Each composite sample is subjected to $\sim 4 \mathrm{GPa}$ average compressive loading. As the interface count increases with volume fraction, the number of reflections and stress concentrations can result in local damage. Interface separation and local tension resulting in the opening of voids introduces additional heterogeneity, further increasing the experimentally observed rise time.

The CTH simulation parameters are not intended to replicate the effects of interface separation and void growth. As the interfaces are assumed to stay welded, the simulations underestimate the level of heterogeneity and scattering efficiency, and therefore underestimate the observed rise time. The additional damage also inhibits the passage of secondary reflections catching up with the lead shock wave. Overshoot is not observed in the largest bead diameters in the $40 \%$ glass by volume composites.
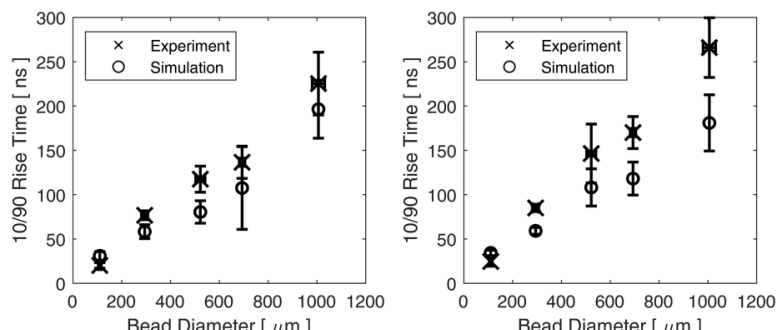

FIG. 11. Rise time as a function of bead diameter for $30 \%$ glass (left) and $40 \%$ glass (right) by volume composites. Both the experimental and simulation results are shown.
The linear scaling with bead size is consistent across the entire glass volume fraction range under examination. Smaller beads approach the unmodified shock thickness in the matrix material as seen in the prior work on tungsten carbide and alumina filled epoxy composites. ${ }^{15}$ The linear regime will also break down at larger bead sizes due to the rapid decrease in number of interfaces per volume as the number of beads becomes fewer. In this regime, there will be regions of significant shock disruption immediately next to regions of strong sharp shock. The increasing magnitude of the error bounds on shock rise time with larger beads begins to indicate the departure from the linear shock rise time scaling regime. The high level of spatial heterogeneity at the largest bead sizes also suggests that the assumption of a steady shock wave is beginning to break down. As the spacing in-between particulates increases, the shock does not remain disrupted and its passage through homogeneous regions of matrix material results in steepening of the shock front, as demonstrated in Fig. 6 (right).

When examined in light of the suggested linear relationship between the shock rise time and mean particle diameter (Fig. 11), the shock speed appears to be an appropriate scale factor. When the rise time is multiplied by the shock speed (assuming a steady wave), the length scale that is computed is nearly equal to the mean bead diameter present in the composite. Therefore, measuring the bulk shock speed and knowing something about the dominant composite length scale can give an estimate of the level of shock front disruption due to scattering by randomly dispersed composites. The shock speed of the composite contains in it information about the impedance mismatch as well as the material properties. A simple dimensional analysis suggests a scale factor with units of inverse velocity, as shown below for the rise time $\left(\tau_{\text {rise }}\right)$ in terms of the bead diameter $\left(d_{p}\right)$ and shock speed in the composite $\left(U_{s}\right)$ :

$$
\tau_{\text {rise }}=f\left(v_{p}\right) \frac{d_{p}}{U_{S}}
$$

where $f\left(v_{p}\right)$ is a function of the volume fraction of the particles $v_{p}$. The functional form of the $f$ has not been determined in the present study, but, for the volume fraction of particles considered in this study (30\% and $40 \%$ ), it appears to be an increasing function of the volume fraction of the particles in the composite.

The uncertainties associated with the rise time measurements increase as the bead size increases. For the smallest bead size, the uncertainty is on the order of the shock width, while it is larger for the biggest bead size (Fig. 11). Nonuniformity of the microstructure is a function of bead size as there are fewer large beads than small ones for a given volume fraction. Hence, the spacing between the beads in larger bead size composites is more varied. The variation in rise time, which is indicated by the uncertainty, can be viewed a measure of the nonuniformity.

\section{Shock disruption in bimodal composites}

A series of experiments was conducted and simulated to determine the level of shock front modification that can be achieved in bimodal composites with beads of two different bead sizes. An experimental matrix was designed using the $30 \%$ glass by volume single mode as the base and $40 \%$ glass by volume single mode bead diameter observations as a basis for comparison. Each bimodal composite 
consists of a base bead mix at $30 \%$ by volume, with smaller diameter interstitial beads added in increments of $10 \%$ glass by volume. Rise times are compared for $40 \%$ glass by volume single mode and bimodal composites. Three base bead mixes were chosen for this study: $500 \mu \mathrm{m}, 700 \mu \mathrm{m}$, and $1000 \mu \mathrm{m}$ at $30 \%$ by volume. To these base mixes, $100 \mu \mathrm{m}, 300 \mu \mathrm{m}, 500 \mu \mathrm{m}$, and $700 \mu \mathrm{m}$ diameter interstitial beads were added. Simulation results indicated that the addition of smaller beads than the base mix as interstitials reduced shock wave rise time by an appreciable amount. This trend persisted in composites with a large diameter difference between the base and the interstitial. The trend was not nearly as strong when base and interstitial beads were similar in size. Increasing composite heterogeneity resulting in decreasing shock thickness was an unexpected outcome.

For reference, a horizontal line has been added to each plot showing the rise time associated with a single mode base bead size, $500 \mu \mathrm{m}$ (Fig. 12) and $1000 \mu \mathrm{m}$ (Fig. 13) for $40 \%$ glass by volume. This will allow for evaluating whether or not a bimodal distribution is a more effective shock front disrupter than a simple single mode bead diameter distribution composite. Furthermore, it shows how much more effective increasing the glass volume fraction is at the shock front disruption. Reported experimental rise times and uncertainties are based on three particle velocity traces on the rear surface of a single target. This ensures that the rise time uncertainty is due solely to spatial heterogeneity effects, as opposed to subtle changes in impact velocities. All measured impact velocities were between $1000 \mathrm{~m} / \mathrm{s}$ and $1010 \mathrm{~m} / \mathrm{s}$.

The first bimodal composites examined consist of $500 \mu \mathrm{m}$ at $30 \%$ by volume glass as the base material, with addition of 100 and $300 \mu \mathrm{m}$ interstitial beads. Rise times from the experimental observations are shown in Fig. 12. The results show an increase in rise time with the addition of smaller particulates up to a total of $50 \%$ glass by volume. The use of smaller interstitial particles resists the tendency of the shock front to steepen as it otherwise would when passing through the large regions in between the base beads. The composite shock rise time response behaves in a similar fashion to simply adding more particulates of a single size. As heterogeneity is increased, sharp localized reflection interaction and tension become more significant, and interface degradation may begin to play a larger role.

Figure 13 shows the rise time dependence of a $1000 \mu \mathrm{m}$ base mix at $30 \%$ glass by volume with either $300 \mu \mathrm{m}$ or $500 \mu \mathrm{m}$
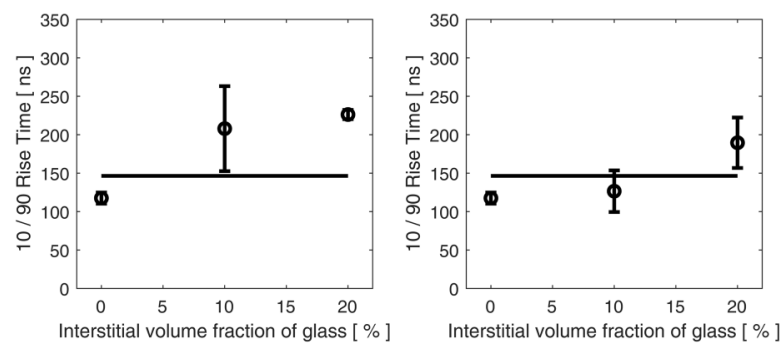

FIG. 12. Experimentally observed shock rise times for $30 \%$ volume fraction $500 \mu \mathrm{m}$ glass beads base with $100 \mu \mathrm{m}$ (left) and $300 \mu \mathrm{m}$ (right) interstitial glass beads in a PMMA matrix. The horizontal line is the rise time for $40 \%$ glass volume fraction composite with single mode $500 \mu \mathrm{m}$ glass beads.
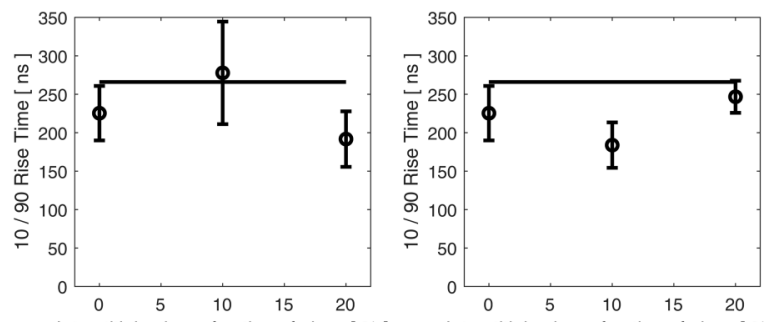

Interstitial volume fraction of glass [\% ]

Interstitial volume fraction of glass [ \% ]

FIG. 13. Experimentally observed shock rise times for $30 \%$ volume fraction $1000 \mu \mathrm{m}$ glass beads base with $300 \mu \mathrm{m}$ (left) and $500 \mu \mathrm{m}$ (right) interstitial glass beads in a PMMA matrix. The horizontal line is the rise time for $40 \%$ glass volume fraction composite with single mode $1000 \mu \mathrm{m}$ glass beads.

interstitials. While the bimodal $500 \mu \mathrm{m}$ base composites demonstrate that rise time increases with the addition of smaller interstitials to some degree, the $1000 \mu \mathrm{m}$ bimodal composites do not show significant rise time increases above the $40 \%$ glass by volume single mode case. Rise times appear to approach a maximum or saturated value. Moving to larger and larger beads will not produce a significant change in rise time, as the spacing between beads increases with increasing mean diameter. It is possible that a higher volume fraction of smaller interstitial beads is required to affect an increase in the shock rise time in composites with a large mean diameter dominating base mix. The $1000 \mu \mathrm{m}$ beads may be approaching the limit of scattering effectiveness, as postulated by Rayleigh for elastic scattering. ${ }^{8}$ Much the same as having inclusions of a smaller/similar diameter as the shock thickness in the pure matrix material reduces scattering effectiveness, having oversize particulates may also result in reduced scattering efficiency, as the tendency for shocks to steepen in homogeneous materials is not balanced by reflections at interfaces.

\section{SUMMARY AND CONCLUSIONS}

Examination of the shock wave structure as a function of microstructure in particulate composites similar to engineered heterogeneous solids (e.g., concrete, energetic materials) was undertaken to determine the effects of particle size and density on scattering effectiveness and shock front thickening. A model particulate composite, with an impedance mismatch ratio falling within the range bounded by energetic materials on the low end and concretes on the high end, was developed to match interface bonding conditions of the engineering materials of interest and provide a simple, repeatable geometry to model. The model particulate composite consisted of finely graded glass spheres in a matrix of PMMA polymer and was fabricated using a vacuum assisted compression molding process. Composites were fabricated with a variety of bead sizes (100-$1000 \mu \mathrm{m}$ ), with a glass bead loading of $30 \%$ and $40 \%$. Normal plate impact experiments along with heterodyne velocimetry were used to characterize the shock structure in the composites.

Shock hydrocode simulations were undertaken to determine the appropriate bead diameters for exploration, the degree of monodispersity required for consistent results, and to confirm that the structured waves were steady. Experiments were then conducted with single mode, and bimodal bead diameter distributions and the 
performance were evaluated by using the shock wave rise time as a metric. For a given volume fraction of the glass particulates, the shock Hugoniot was found to be independent of the particle size. Statistics were generated by using free surface velocity measurements at multiple points on the rear surface of each composite target to account for spatial heterogeneity in shock response due to the random placement of particulates. The strain rate in the shock front was found to scale as the square of the shock stress, which is similar to observations on other heterogeneous materials such as layered solids and granular materials. Shock rise time was found to scale linearly with the particle diameter divided by the shock wave speed for composites with monodisperse particle diameter distributions. This simple one-parameter model may be used to predict shock rise times for a variety of materials since the mechanical mismatch and impact velocity information are contained within the Lagrangian shock speed. The rise times for the large particle size composites are found to be an order of magnitude or more than that of the pure matrix. Experiments conducted on composites with a bimodal distribution of the size of particles revealed that the rise time could be significantly increased with certain combinations of particles sizes through enhancement of dispersion.

\section{ACKNOWLEDGMENTS}

The support of the Air Force Office of Scientific Research and Air Force Research Laboratory through the University Center of Excellence in High-Rate Deformation Physics of Heterogeneous Materials (Award No. FA9550-12-1-0091) is gratefully acknowledged. M. B. Rauls acknowledges the support from the Department of Defense (DoD) through the National Defense Science and Engineering Graduate Fellowship (NDSEG) program. We thank Dr. Christopher (Kit) Neel and Professor John Borg for stimulating discussions and help during the study.

\section{APPENDIX: SUMMARY OF EXPERIMENTAL PARAMETERS AND RESULTS}

The experimental parameters and the measured and computed quantities for all the experiments discussed in this paper are presented in Table IV.

TABLE IV. Experimental parameters and results.

\begin{tabular}{|c|c|c|c|c|c|c|c|c|}
\hline $\begin{array}{l}\text { Experiment } \\
\text { No. }{ }^{a}\end{array}$ & $\begin{array}{c}\text { Sample } \\
\text { composition }\end{array}$ & $\begin{array}{c}\text { Sample } \\
\text { thickness } \\
(\mathrm{mm})\end{array}$ & $\begin{array}{c}\text { Impact } \\
\text { velocity } \\
(\mathrm{m} / \mathrm{s})\end{array}$ & $\begin{array}{c}\text { Shock } \\
\text { speed } \\
(\mathrm{mm} / \mu \mathrm{s})\end{array}$ & $\begin{array}{c}\text { Free surface } \\
\text { particle velocity } \\
(\mathrm{m} / \mathrm{s})\end{array}$ & $\begin{array}{c}10-90 \\
\text { rise } \\
\text { time (ns) }\end{array}$ & $\begin{array}{l}\text { Shock } \\
\text { stress } \\
(\mathrm{GPa}) \\
\end{array}$ & $\begin{array}{l}\text { Strain } \\
\text { rate } \\
(1 / s) \\
\end{array}$ \\
\hline 1 & Pure PMMA & 7.10 & 781.4 & 3.59 & 1216 & 5 & 2.61 & $2.48 \times 10^{7}$ \\
\hline 2 & Pure PMMA & 7.06 & 1011.3 & 3.82 & 1547 & 5 & 3.64 & $3.09 \times 10^{7}$ \\
\hline 3 & Pure PMMA & 7.09 & 1211.6 & 4.00 & 1842 & 5 & 4.50 & $3.59 \times 10^{7}$ \\
\hline 4 & $100 / 30$ & 7.05 & 1006.1 & 3.85 & 1422.2 & 20 & 4.58 & $7.32 \times 10^{6}$ \\
\hline 5 & $300 / 30$ & 6.92 & 1019.6 & 3.98 & 1437 & 77 & 4.68 & $1.88 \times 10^{6}$ \\
\hline 6 & $500 / 30$ & 7.07 & 1008.6 & 3.99 & 1459.7 & 118 & 4.31 & $1.25 \times 10^{6}$ \\
\hline 7 & $700 / 30$ & 7.07 & 1009.0 & 3.99 & 1476.2 & 137 & 4.18 & $1.08 \times 10^{6}$ \\
\hline 8 & $1000 / 30$ & 7.04 & 1009.5 & 3.94 & 1480 & 225 & 4.16 & $6.66 \times 10^{5}$ \\
\hline 9 & $100 / 40$ & 4.96 & 1006.7 & 4.10 & 1408.6 & 34 & 4.70 & $4.05 \times 10^{6}$ \\
\hline 10 & $100 / 40$ & 7.10 & 1078.4 & 4.03 & 1496.6 & 25 & 5.16 & $6.04 \times 10^{6}$ \\
\hline 11 & $100 / 40$ & 10.00 & 1014.2 & 3.61 & 1407 & 28 & 4.84 & $5.65 \times 10^{6}$ \\
\hline 12 & $300 / 40$ & 7.06 & 1025.1 & 4.03 & 1436 & 85 & 4.78 & $1.68 \times 10^{6}$ \\
\hline 13 & $500 / 40$ & 4.85 & 1002.9 & 3.95 & 1509.2 & 78 & 3.81 & $1.96 \times 10^{6}$ \\
\hline 14 & $500 / 40$ & 7.07 & 1017.3 & 4.18 & 1415 & 146 & 4.82 & $9.25 \times 10^{5}$ \\
\hline 15 & $500 / 40$ & 9.90 & 1000.0 & 3.96 & 1470.8 & 96 & 4.08 & $1.54 \times 10^{6}$ \\
\hline 16 & $500 / 40$ & 7.62 & 580.8 & 3.78 & 861 & 270 & 2.25 & $3.37 \times 10^{5}$ \\
\hline 17 & $500 / 40$ & 7.62 & 787.4 & 4.00 & 1113 & 124 & 3.53 & $9.00 \times 10^{5}$ \\
\hline 18 & $500 / 40$ & 7.62 & 1379.3 & 4.24 & 2049 & 51 & 5.20 & $3.89 \times 10^{6}$ \\
\hline 19 & $700 / 40$ & 7.05 & 1010.5 & 4.22 & 1408.9 & 170 & 4.76 & $7.85 \times 10^{5}$ \\
\hline 20 & $1000 / 40$ & 7.08 & 1012.1 & 4.09 & 1481 & 266 & 4.19 & $5.45 \times 10^{5}$ \\
\hline 21 & $500 / 30+100 / 10$ & 7.12 & 1007.1 & 4.07 & 1404 & 208 & 4.74 & $6.64 \times 10^{5}$ \\
\hline 22 & $500 / 30+100 / 20$ & 7.17 & 1009.8 & 4.13 & 1480 & 226 & 4.16 & $6.34 \times 10^{5}$ \\
\hline 23 & $500 / 30+300 / 10$ & 7.00 & 1004.6 & 3.99 & 1417 & 127 & 4.59 & $1.12 \times 10^{6}$ \\
\hline 24 & $500 / 30+300 / 20$ & 7.05 & 1004.9 & 4.15 & 1374 & 190 & 4.96 & $6.98 \times 10^{5}$ \\
\hline 25 & $1000 / 30+300 / 10$ & 7.06 & 1008.3 & 4.17 & 1462 & 278 & 4.28 & $5.05 \times 10^{5}$ \\
\hline 26 & $1000 / 30+300 / 20$ & 6.93 & 1010.8 & 3.93 & 1458 & 192 & 4.36 & $7.74 \times 10^{5}$ \\
\hline 27 & $1000 / 30+500 / 10$ & 7.07 & 1013.3 & 4.16 & 1507 & 184 & 4.00 & $7.89 \times 10^{5}$ \\
\hline 28 & $1000 / 30+500 / 20$ & 7.09 & 1014.4 & 4.19 & 1520 & 247 & 3.91 & $5.88 \times 10^{5}$ \\
\hline
\end{tabular}

${ }^{a}$ The experiments are numbered in the table for convenience and do not represent the order in which they were conducted. 
Experiments 4-20 were conducted on composites with sample composition, $\mathrm{S} / \mathrm{P}$, with a monodispersed $\mathrm{P}$ volume fraction in percentage of glass beads with diameter $\mathrm{S}$ in $\mu \mathrm{m}$. Experiments $21-28$ were conducted on bimodal composites with sample composition, $\mathrm{S}_{1} / \mathrm{P}_{1}+\mathrm{S}_{2} / \mathrm{P}_{2}$, where $\mathrm{P}_{1}$ and $\mathrm{P}_{2}$ are the volume fraction in percentage of glass beads with diameters $S_{1}$ and $S_{2}$ in $\mu \mathrm{m}$, respectively.

Experiments 1-3 (Fig. 4) were conducted on pure PMMA for characterizing its Hugoniot [Eq. (5)]. Experiments 9-11 and 13-15 (Fig. 7) were conducted to investigate the steady wave nature of shocks in particulate composites. Experiments 14 and 16-18 were used to establish the Hugoniot of the $40 \%$ volume fraction $500 \mu \mathrm{m}$ glass beads composite [Fig. 8 and Eq. (6)].

The shock stress was computed using the measured impact and the free surface velocities together with the known density and Hugoniot for the impactor material, 6061-T6 aluminum (Table I). The strain rate associated with the shock front was calculated using Eq. (8).

\section{REFERENCES}

'M. A. Meyers, Dynamic Behavior of Materials (John Wiley, New York, 1994).

${ }^{2}$ L. Davison, Fundamentals of Shock Wave Propagation in Solids (Springer, Berlin, 2008).

${ }^{3}$ D. E. Grady, J. Mech. Phys. Solids 46, 2017 (1998).

${ }^{4}$ S. Zhuang, G. Ravichandran, and D. E. Grady, J. Mech. Phys. Solids 51, 245 (2003).

5. W. Swegle and D. E. Grady, J. Appl. Phys. 58, 692 (1985).

${ }^{6}$ A. Molinari and G. Ravichandran, J. Appl. Phys. 95, 1718 (2004).

${ }^{7}$ A. Molinari and G. Ravichandran, J. Mech. Phys. Solids 54, 2495 (2006).

${ }^{8}$ D. E. Grady, J. Appl. Phys. 107, 013506 (2010).

${ }^{9}$ R. E. Setchell and M. U. Anderson, J. Appl. Phys. 97, 083518 (2005).

${ }^{10} \mathrm{M}$. B. Rauls and G. Ravichandran, "Shock wave structure in particulate composites,” Procedia Eng. 103, 515 (2015).

${ }^{11}$ M. B. Rauls, "Shock wave behavior of particulate composites," Ph.D. thesis (California Institute of Technology, 2015).
12J. L. Jordan, E. B. Herbold, G. Sutherland, A. Fraser, J. Borg, and D. W. Richards, J. Appl. Phys. 109, 013531 (2011).

${ }^{13}$ C. Neel and N. N. Thadhani, AIP Conf. Proc. 1195, 1257 (2009).

${ }^{14}$ C. Neel and N. N. Thadhani, J. Appl. Phys. 109, 013511 (2011).

${ }^{15}$ T. J. Vogler, C. S. Alexander, J. L. Wise, and S. T. Montgomery, J. Appl. Phys. 107, 043520 (2010).

${ }^{16}$ R. E. Setchell, M. U. Anderson, and S. T. Montgomery, J. Appl. Phys. 101, 083527 (2007).

17J. C. F. Millett, N. K. Bourne, J. Akhavan, and A. M. Milne, J. Appl. Phys. 97, 043524 (2005).

${ }^{18}$ K. Tsembelis, W. G. Proud, G. R. Willmott, and D. L. A. Cross, AIP Conf. Proc. 706, 1488 (2004).

19. J. Dick, A. R. Martinez, and R. S. Hixson, in Proceedings of the 11th Symposium on Detonation (Office of Naval Research, 1998), p. 317.

${ }^{20}$ D. E. Munson, R. R. Boade, and K. W. Schuler, J. Appl. Phys. 49, 4797 (1978).

${ }^{21}$ T. J. Vogler, J. P. Borg, and D. E. Grady, J. Appl. Phys. 112, 123507 (2012).

${ }^{22}$ M. R. Baer, Thermochim. Acta 384, 351 (2002).

${ }^{23}$ J. L. Jordan, L. Ferranti, R. A. Austin, R. D. Dick, J. R. Foley, N. N. Thadhani, D. L. McDowell, and D. J. Benson, J. Appl. Phys. 101, 093520 (2007).

${ }^{24}$ E. B. Herbold, V. F. Nesterenko, D. J. Benson, J. Cai, K. S. Vecchio, F. Jiang, J. W. Addiss, S. M. Walley, and W. G. Proud, J. Appl. Phys. 104, 103903 (2008).

${ }^{25}$ L. M. Barker and R. E. Hollenbach, J. Appl. Phys. 41, 4208 (1970).

${ }^{26}$ J. A. Brydson, Plastics Materials (Butterworth-Heinemann, Oxford, 1999).

${ }^{27}$ P. D. Washabaugh, "An experimental investigation of mode-I crack tip deformation," Ph.D. thesis (California Institute of Technology, 1990).

${ }^{\mathbf{2 8}}$ D. H. Dolan, Rev. Sci. Instrum. 81, 053905 (2010).

${ }^{29}$ See http://www.sandia.gov/CTH/ for "CTH Reference Manuals."

${ }^{30} \mathrm{E}$. S. Hertel et al., Shock Waves @ Marseille I (Springer, Berlin, 1995), p. 377.

${ }^{31}$ J. C. Russ and R. T. Dehoff, Practical Stereology (Kluwer Academic, Dordrecht, 2000).

${ }^{32}$ S. P. Lyon and J. D. Johnson, "SESAME: The Los Alamos National Laboratory Equation of State Database," Report No. LA-UR, 92-0307, 1993.

${ }^{33}$ G. I. Kerley, "CTH Equation of State Database MGRUN Option," Report No. KPS00-4, Kerley Publishing Services, Albuquerque, NM, 2000.

${ }^{34}$ D. Lacina, C. Neel, and D. Dattelbaum, J. Appl. Phys. 123, 185901 (2018).

${ }^{35}$ D. R. Bland, IMA J. Appl. Math. 1, 56 (1965). 